\title{
Too big or too small?
}

\author{
Carl L. Backer, $\mathrm{MD},{ }^{\mathrm{a}}$ and Luciana Young, $\mathrm{MD}^{\mathrm{b}}$
}

\footnotetext{
From the Divisions of a Cardiovascular-Thoracic Surgery and ${ }^{\mathrm{b} C a r d i o l o g y}$, Ann \& Robert H. Lurie Children's Hospital of Chicago, Chicago, Ill.

Disclosures: Authors have nothing to disclose with regard to commercial support.

Received for publication Feb 24, 2016; accepted for publication Feb 25, 2016; available ahead of print March 25, 2016.

Address for reprints: Carl L. Backer, MD, Division of Cardiovascular-Thoracic Surgery, Ann \& Robert H. Lurie Children's Hospital of Chicago, 225 E Chicago Ave, mc 22, Chicago, IL 60611 (E-mail: cbacker@ luriechildrens.org).

J Thorac Cardiovasc Surg 2016;151:1547-8

$0022-5223 / \$ 36.00$

Copyright (c) 2016 by The American Association for Thoracic Surgery

http://dx.doi.org/10.1016/j.jtcvs.2016.02.059
}

Dr Hasegawa and colleagues ${ }^{1}$ from Kobe Children's Hospital have demonstrated the predictive value of diastolic retrograde flow in the descending aorta for identifying high-flow systemic-to-pulmonary artery shunts before patients develop cardiogenic shock from pulmonary overcirculation. The authors calculated the ratio of the mean diastolic retrograde flow velocity time interval (VTI) to the mean systolic antegrade flow VTI in the descending thoracic aorta. Analysis of the outcomes of 53 patients indicated that a ratio $>0.80$ was correlated with the occurrence of major postoperative adverse events related to high-flow shunting.

Historically, the issue of appropriate shunt size has been critical for surgeons. If the shunt is too small, pediatric patients remain cyanotic and the shunts put them at risk for thrombosis. Conversely, if the shunt is too big, children are at risk for pulmonary overcirculation leading to congestive heart failure, necrotizing enterocolitis, coronary insufficiency, and possibly cardiac arrest. The simple, but effective, strategy of assessing shunt flow is a valuable tool, one to be placed in the armamentarium of surgeons who perform shunt procedures. Ideally, this assessment would be done in real time, in the operating room, as soon as the clamps are released from the shunt, so that appropriate action could be taken immediately.

Doppler echocardiography is a useful tool for assessing the diastolic "steal" phenomenon in patients who have cardiovascular lesions resulting in prominent diastolic reversal of flow in the abdominal aorta, such as significant aortic insufficiency, patent ductus arteriosus, arteriovenous malformations, and aortopulmonary window. The ratio of flow reversal in proportion to forward flow in the descending aorta, when used in conjunction with arterial oxygen saturation, has been shown to be correlated strongly ${ }^{2}$ with the ratio of pulmonary blood flow to systemic blood flow (Qp/Qs). The higher the diastolic flow ratio, the higher the Qp/Qs. Hasegawa and colleagues ${ }^{1}$ have added significance to their measurements by defining an absolute value that can be used to predict which patients are at risk for adverse consequences related to substantial pulmonary overcirculation.

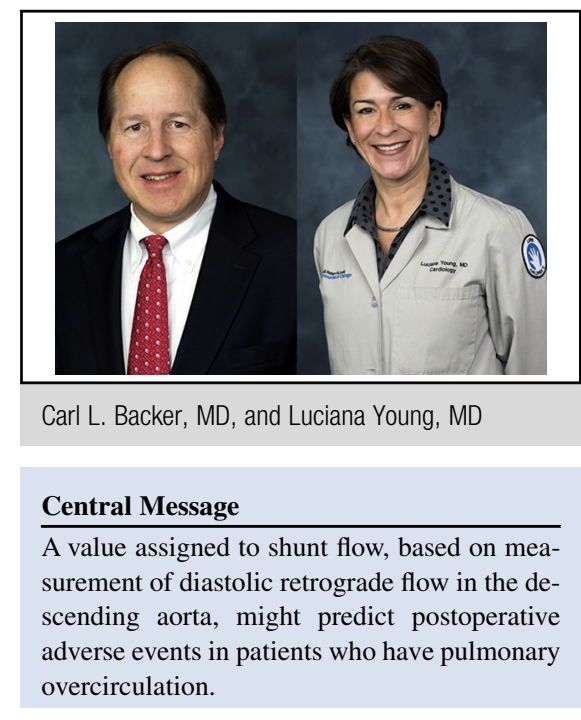

See Article page 1540.

Certain limitations accompany this technique. The series used is relatively small $(n=53)$, and only 4 patients had major adverse events. The diastolic flow reversal ratio represents an index that only reflects $\mathrm{Qp} / \mathrm{Qs}$ and is not a direct measurement. Secondary sources of pulmonary blood flow, such as aortopulmonary collaterals, prograde flow across the pulmonary artery, and the presence of a residual shunt, such as a patent ductus arteriosus, may confound the calculation and therefore render it inaccurate. A suboptimal angle of insonation may affect the adequacy of the Doppler signal. Because of these limitations, the cardiac care team needs to monitor corroborating parameters, such as mixed venous $\mathrm{O}_{2}$ saturation, lactate, urine output, etc.

The transesophageal echocardiogram would be very helpful in assessing these parameters with the patient still in the operating room. Unfortunately, imaging of the descending aorta to obtain an adequate Doppler signal may be challenging using transesophageal echocardiography, because the vessel is usually perpendicular to the Doppler cursor. We could consider doing transthoracic imaging from the abdominal view, if needed, but the physiologic changes that occur when patients are under general anesthesia and mechanically ventilated would need to be taken into consideration.

The authors are to be congratulated on promoting a tool that is available to all critical care units and, potentially, to operating rooms. Use of this tool may help diminish the number of patients who have excessive pulmonary blood flow and its attendant well known risks of heart 
failure, necrotizing enterocolitis, and coronary ischemia. This tool may help us create shunts that are neither too small nor too big, but rather "just right." This study will enhance our postoperative management of the delicate balance between pulmonary and systemic blood flow in patients who receive aortopulmonary shunts, to improve clinical outcomes.

\section{References}

1. Hasegawa T, Oshima Y, Tanaka T, Maruo A, Matsuhisa H. Clinical assessment of diastolic retrograde flow in the descending aorta for high-flow systemic-to-pulmonary artery shunting. J Thorac Cardiovasc Surg. 2016; 151:1540-6.

2. Rychik J, Bush DM, Spray TL, Gaynor JW, Wernovsky G. Assessment of pulmonary/systemic blood flow ratio after first-stage palliation for hypoplastic left heart syndrome: development of a new index with the use of Doppler echocardiography. J Thorac Cardiovasc Surg. 2000;120:81-7. 